\title{
Conocimiento didáctico del contenido curricular en química: la selección de contenidos para enseñar el concepto de estructura en quimica orgánica
}

\author{
Raquel Jurado Arcos ${ }^{1}$ y Diana Lineth Parga $L^{2}$.
}

${ }^{1}$ Candidata a Magíster en Docencia de la Química. Universidad Pedagógica Nacional. Profesora de Ciencias Naturales del Distrito Especial de Bogotá. ${ }^{2}$ Profesora del Departamento de Química. Universidad Pedagógica Nacional. juradoarcos2000@gmail.com, dparga@pedagogica.edu.co

\section{Resumen}

El presente comunicado muestra los resultados de una investigación realizada para optar al título de magister en Docencia de la Química. Se pretendió caracterizar los aspectos que tiene en auenta el profesorado en ejercicio auando seleccionan y diseñan los contenidos de enseñanza en química orgánica. La teoría estructural es la base para la enseñanza de la química orgánica, por ello otro de los propósitos fue indagar si el profesorado tiene en cuenta la teoría dual y la teońa unitaria y los aportes que éstas hacen para el desarrollo de la teoría estructural en el momento de enseñar la química orgánica.

\section{Palabras claves}

Conocimiento didáctico del contenido curricular en química (CDCCQ), Contenidos conceptuales, procedimentales y actitudinales, Teoría Dual, Teoría Unitaria, Teoría Estructural.

\section{Conocimiento Didàctico del Contenido Curricular}

El trabajo se enmarca en la línea de investigación Didáctica de los contenidos curriaulares en química (DCCQ), bajo el proyecto de investigación: Conocimiento didáctico del contenido curricular en química y el diseño de tramas conceptuales, liderado por el grupo investigador Altemativas para la enseñanza de las ciencias naturales ALTERNACIENCIAS, de la Universidad Pedagógica Nacional. Dicha línea de investigación está emparentada con las líneas de investigación intemacionales: The Knowledge growth in teaching, Subject-matter knowledge, curricular knowledge (Parga y Martínez, 2006). con:

En el presente trabajo se asumirán los planteamientos de Parga y Martínez (2006) en relación

$>$ Los contenidos curriculares se asumen desde una perspectiva compleja y crítica, y cómo éstos funcionan en el aula.

$>$ El profesional docente es un agente diseñador de los contenidos curriculares y su práctica dentro del aula.

> El desarrollo profesional está en relación con la investigación en el aula y en particular de los contenidos de enseñanza.

$>$ El ejercicio didáctico de la selección y desarrollo de contenido curricular está basado en un análisis histórico y epistemológico, que permite valorar dichos contenidos con la sociedad, la tecnología y el ambiente.

$>$ Las tramas conceptuales, como productos de la investigación, contribuyen a organizar el conocimiento, articularlo en unidades didácticas, y poner de manifiesto las interrelaciones entre los contenidos; ofreciendo una visión de conjunto de un determinado concepto y sus relaciones con otros. 
> Las unidades didácticas son módulos de programación, diseño y desarrollo de la enseñanza que permiten organizar los programas escolares dotándolos de capacidad para integrar contenidos diversos y de estructurar períodos relativamente largos de la vida escolar.

La selección de contenidos a enseñar es una decisión que debe tomar el docente en su desarrollo profesional. Esta selección debe estar de acuerdo con el contexto escolar donde ejerce su labor, con el alumnado y con la disciplina a enseñar, por esto es indispensable que el maestro reflexione al momento de elegirlos teniendo en cuenta que es una decisión política puesto que todo lo que se hace en el aula de dase está mediado o tiene que ver con ella. Los criterios que siguen los docentes para seleccionar los contenidos son disciplinares, no constituyen saberes estáticos, ya acabados, sino problemas a los cuales enfrentarse en busca de una solución. El currículo se organiza en tomo a preguntas más que a respuestas. Por ello, cabe pensar que la propia historia de las ciencias debe desempeñar un papel esencial en la organización y secuenciación de los contenidos.

\section{Objetivos}

Al enseñar la química orgánica es indispensable conocer la teonía estructural como una teoría central. Son propósitos de esta investigación:

$>$ Caracterizar los criterios que tiene en cuenta el profesorado cuando selecciona los contenidos al enseñar el concepto de estructura en química orgánica.

> Establecer la importancia que da el profesorado al concepto de estructura en la química orgánica y si tienen en cuenta los criterios cualitativos de la teoría dual y la teoría unitaria al enseñar

> Determinar cómo se afecta el diseño aurriaular al incluir el conocimiento histórico epistemológico, el conocimiento psicopedagógico, conocimiento disciplinar y el contexto escolar.

\section{Metodología}

El marco metodológico en el cual se desarrolló el presente trabajo es de tipo cualitativo ya que ésta orienta y describe los fenómenos sociales y por consiguiente los educativos y se interesa por el estudio de los significados y acciones humanas desde la perspectiva de los mismos agentes sociales. Por lo tanto, es pertinente abordar esta metodología para llevar a cabo la caracterización de la selección de contenidos en profesores en ejercicio, dado el contexto educativo que se analizó (Villamizar, 2008).

La investigación se fundamentó desde en una perspectiva interpretativa, pues indagó si las profesoras en ejercicio de un Colegio Distrital de Bogotá, utilizaban el concepto de estructura para enseñar la química orgánica y los criterios que tienen para seleccionar los contenidos a enseñar.

Se trabajó con cuatro profesoras en ejercicio del aérea de Ciencias Naturales. A las participantes se les informó sobre los aspectos más relevantes de la investigación especialmente sobre la importancia y los propósitos de la misma. Se hizo especial énfasis en la confidencialidad de los datos, utilizando para tal fin números asignados para cada una de las profesoras.

Los instrumentos utilizados fueron: encuestas, entrevistas semiestructuradas; se hizo el análisis documental de los cuademos del estudiantado y de los libros de texto utilizados por las profesoras y sus materiales curriculares (los que ellas diseñaron). 
El diseño de encuestas fue para reunir información que permitiera determinar qué tienen en cuenta las profesoras cuando seleccionan los contenidos para enseñar el concepto de estructura en química orgánica, y si utilizan en su explicación los postulados de la teoría dual y la teoría unitaria en la enseñanza de la química orgánica, teniendo en cuenta los citerios aualitativos propios del siglo XIX donde se dio la rivalidad entre estas dos teorías.

En las enquestas y entrevistas se induyeron preguntas para determinar cómo se afecta el diseño aurriaular al induir el conocimiento histórico epistemológico, el conocimiento psicopedagógico, conocimiento disciplinar y el contexto escolar (Mora y Parga, 2008).

Para aplicar la entrevista del instrumento tres relacionada con "Conocimiento Didáctico del Contenido Curriaular en Química" Criterios de Selección de Contenidos, se escogieron las dos profesoras con mayor experiencia en la enseñanza de la química orgánica.

También se hizo la revisión de documentos de las profesoras de química orgánica. Se revisaron auatro libros de texto utilizados por las profesoras en ejercicio y tres cuademos de estudiantes seleccionados al azar en el grado once del Colegio de Bogotá. De igual forma se revisaron documentos del Colegio, tales como su Proyecto Educativo Institucional, el Plan de área de Ciencias Naturales y Educación Ambiental.

El tratamiento de los instrumentos se llevó a cabo por medio de un análisis mixto que involucró un tratamiento de los datos desde un análisis cualitativo, ya que desde este análisis se pretendió darle estructura a los datos, lo aual implicó organizarlos en categorías, unidades y patrones, encontrando sentido a los datos en el marco del planteamiento de problemas .

El análisis de cada una de las respuestas consignadas en las plantillas diseñadas para tal fin, se realizaron teniendo en cuenta algunas categońas y sub-categorías generadas a partir de las conceptualizaciones inicialmente realizadas a través de una codificación abierta, lo que permitió diseñar una matriz de análisis para cada instrumento.

Se establecieron categorías como Altamente Deseable (AD), Deseable (D) Medianamente Deseable (MD), No Deseable (ND) No sabe/ No responde (NS/ NR) No cita (NC) y sub-categorías, las cuales se establecen en una matriz de análisis para cada uno de los instrumentos.

De acuerdo con la naturaleza de la investigación, para caracterizar el conocimiento didáctico del contenido curricular de las profesoras de química de un colegio de Bogotá, en relación con la selección de contenidos a enseñar, y la forma como enseñan el concepto de estructura, se tuvieron en cuenta las categorías de conocimiento y los indicadores referidas por Mora y Parga (2008) y se identificaron así:

\begin{tabular}{|c|c|c|}
\hline $\begin{array}{l}\text { Categorías de } \\
\text { CONOCIMIENTO }\end{array}$ & INDICADOR & $\begin{array}{l}\text { INSTRUMENTO } \\
\text { (se encuentran en } \\
\text { los anexos) }\end{array}$ \\
\hline $\begin{array}{l}\text { Conocimiento disciplinar del } \\
\text { contenido CdC } \\
\text { "Comprender la materia" }\end{array}$ & $\begin{array}{l}\text { 1. Un cuerpo interrelacionado de conceptos. } \\
\text { 2. Teorías y paradigmas de la química. (Teoría dual, } \\
\text { teoría unitaria, teoría estructural). } \\
\text { 3. Este conocimiento determina el diseño curricular. }\end{array}$ & $\begin{array}{l}\text { Instrumento } 1 \\
\text { Instrumento } 2\end{array}$ \\
\hline $\begin{array}{l}\text { Conocimiento Histórico - } \\
\text { Epistemológico. CHE. } \\
\text { "Comprender qué y cómo ha } \\
\text { cambiado el conocimiento" }\end{array}$ & $\begin{array}{l}\text { 1. Mecanismos de producción del conocimiento. } \\
\text { 2. Obstáculos epistemológicos. } \\
\text { 3. Formas de vida de las comunidades científicas. } \\
\text { 4. Debates y controversias, reconstrucciones de } \\
\text { episodios históricos relevantes. } \\
\text { 5. Revoluciones cientúficas y experimentos cruciales. }\end{array}$ & $\begin{array}{l}\text { Instrumento } 4 \\
\text { Instrumento } 5 \\
\text { Instrumento } 6 \\
\text { Instrumento } 7\end{array}$ \\
\hline
\end{tabular}




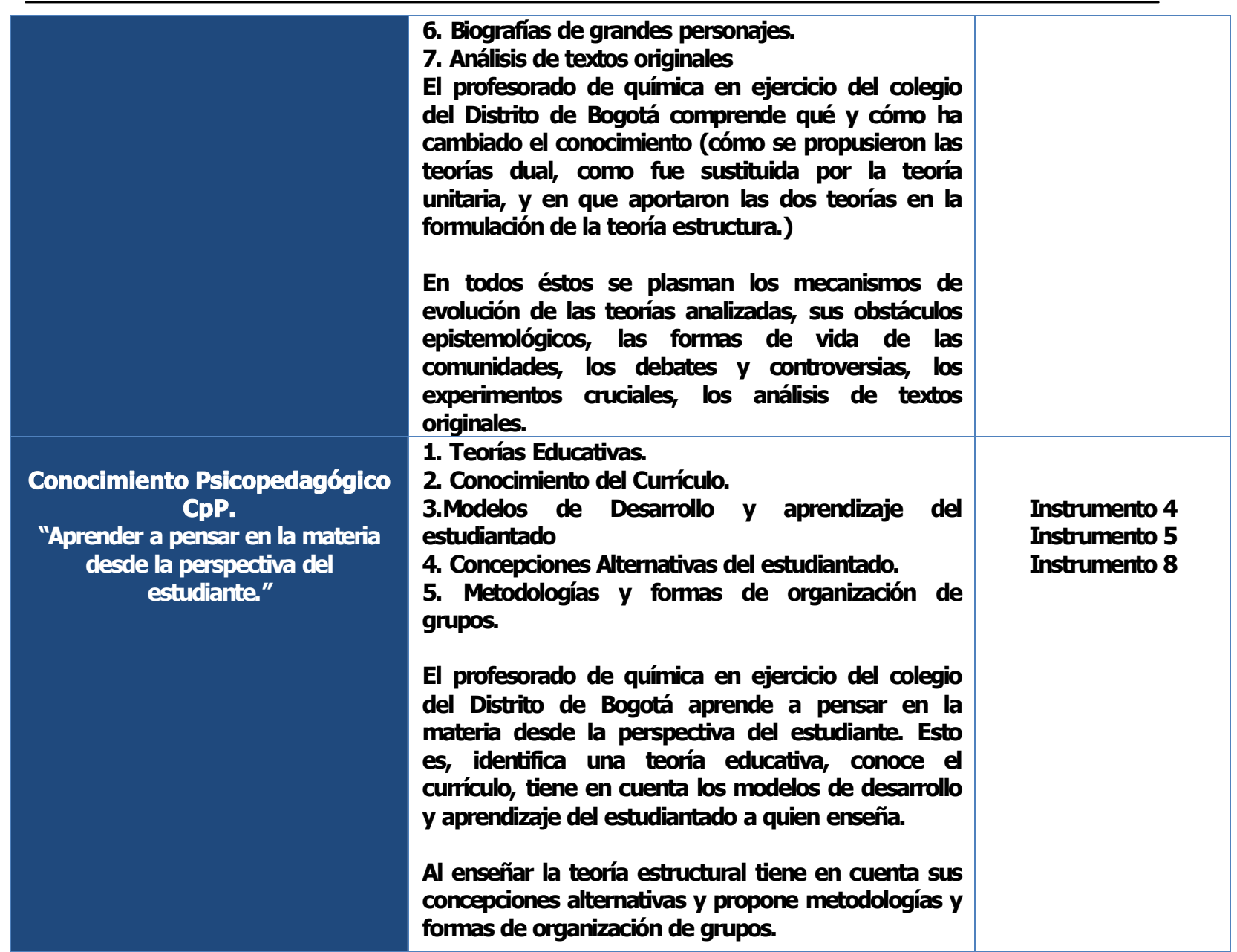

Tabla 1. Indicadores de la investigación.

\section{Conclusiones}

Los aspectos que tienen en auenta las Profesoras en Ejercicio (PE) del Colegio Distrital, para la selección de contenidos a enseñar en química orgánica son prioritariamente los establecidos por el Ministerio de Educación Nacional, quien determina los contenidos a través de los estándares, pero el maestro es quien diseña, organiza y flexibiliza los contenidos dependiendo de la pertinencia.

Las PE mayoritariamente enseñan contenidos conceptuales, sin hacer un registro de contenidos procedimentales y actitudinales. No se mencionan o no son tomados en cuenta.

Con los instrumentos aplicados se recoge la información donde se menciona el conocimiento histórico - epistemológico, pero se evidencia que hay desconocimiento de la historia de la química y cómo se construye el conocimiento en esta disciplina.

Falta apropiación por parte de las PE en relación con las teorías de la química orgánica, por lo tanto no se enseñan. No utiliza el concepto de estructura como fundamental en la química orgánica, no se explica cómo se construye el conocimiento y se imparten una serie de conceptos que son desarticulados y difíiles de comprender por parte del estudiantado.

Se puede afirmar que las profesoras no utilizan el concepto de estructura, ni tampoco utilizan en su explicación los postulados de la teoría dual ni de la teoría unitaria pues los desconocen. 
Es importante el análisis crítico de las dificultades que existen en el grupo de profesores en ejercicio, para adelantar un plan de formación permanente de docentes, con el fin de llenar los vacíos que existen en la química orgánica que se identifican en esta investigación.

Se espera que los PE sean expertos en la selección de contenidos, así como en el diseño de unidades didácticas pues con el ejercicio de la profesión deben mejorar su desempeño, sin embargo, las docentes han diseñado guías y talleres, muchas veces desarticulados y sin la concepción de unidad didáctica, como unidad de programación e hipótesis de trabajo.

Se sugiere trabajar con las docentes en la construcción de tramas conceptuales, utilizando para ello la historia y la epistemología de la química. Pero esto, solo puede ser posible con un profesorado que conozca en profundidad la disciplina a enseñar y que a su vez tenga una formación en didáctica de las ciencias, que utilice el conocimiento psicológico del aprendizaje de la química a la hora de enseñar; que utilice la historia y la epistemología para el diseño y evaluación, curricular, que esté en un proceso permanente de investigación y mejoramiento profesional. Que asuma que enseñar es crear condiciones para producir conocimiento nuevo y por lo tanto requiere una formación continua como investigador y un compromiso con la construcción del conocimiento y de la sociedad.

\section{Bibliográfica}

Ministerio de Educación Nacional. (1998). Lineamientos curriculares. Ciencias Naturales y educación ambiental. Bogotá: Cooperativa editorial magisterio.

Ministerio de Educación Nacional. (2004). Estándares para la excelencia en la educación.

Mora, W. y Parga, D. (2003). Molécula II Ed. Voluntad Bogotá.

Mora, W., Parga, D. (2005). De las investigaciones en preconcepciones sobre mol y cantidad de sustancia, hacia el diseño curricular en química. Educación y pedagogía, Vol. XVII No. 43.

Mora, W. y Parga, D. (2007). Las tramas histórico-epistemológicas en el contexto del conocimiento didáctico del contenido curricular: El diseño de niveles de formulación en la construcción del modelo teórico estructural en la química orgánica. Tecné, Episteme y Didaxis: TED. No. 21. pp. 100-118

Mora, W., Parga, D., (2008). El Conocimiento Didáctico del Contenido Química: Integración de las Tramas de contenido / histórico - epistemológicas con las tramas de Contexto / Aprendizaje. Tecné, Episteme y Didaxis: TED. Número 24.

Mosquera, C. Mora, W. y Garáa, A. (2003). Conceptos fundamentales de la química y su relación con el desamollo profesional del profesorado. Bogotá: Colciencias- Centro de Investigaciones y desarrollo Cient́fico. Universidad Francisco José de Caldas.

Parga, D. y Martínez F. (2006). Conocimiento pedagógico del contenido didáctico de la química y el diseño de tramas conceptuales. Bogotá: Investigación. Universidad Pedagógica Nacional. Departamento de Química.

Parga, D. y Martínez F. (2007). Conocimiento didáctico del contenido curricular en química: una estrategia sustentada en tramas conceptuales. Proyecto de Investigación DQU 025-07. Bogotá: Centro de Investigaciones de la Universidad Pedagógica Nacional.

Torres, L (2004) De las tramas histónicas a las trama didácticas: Una propuesta para el diseño de unidades didácticas en química orgánica. Trabajo de grado de Licenciatura en Química. Bogotá: Universidad Distrital Francisco José de Caldas.

Sánchez B. y Valcárcel M. (2000) ¿Qué tienen en cuenta los profesores cuando seleccionan el contenido de enseñanza? Cambios y dificultades tras un programa de formación. Universidad de Murcia. Enseñanza de las ciencias, 18(3), 423-437.

Satish, C. Kapoor. (1969) The origins of Laurent's Organic Classification- The Isis. University of Chicago Press. 60(4), 477-527 
Shulman, L. (1999). Foreward en Gess - Newsome, J., Lederman, N.G. (Eds),

Examining Pedagogical Content Knowledge. The Construct and its implications for Saience Education. Dordrecht, The Netherlands, Kluwer Academic Publishers, pp. IX - XII. Citado por Parga y Martínez. (2006). Conocimiento Pedagógico del contenido didáctico de la química y el diseño de tramas conceptuales. Proyecto de investigación CIUP.

Stenhouse, L. (1987). Investigación y desarrollo del currículo. Madrid: Editorial Morata.

Valencia, F. (2008). Caracterización del conocimiento didáctico del contenido curricular (CDCC) en los profesores de ciencias al diseñar una unidad didáctica con enfoque ciencia, tecnología, sociedad y ambiente (CTSA). Tesis de maestría en docencia de la química .Universidad Pedagógica Nacional. Bogotá.

Villamizar, D. (2008). Conocimiento didáctico del contenido aurricular en química:

un estudio con profesores en formación inicial. Tesis de grado. Licenciatura en química. Universidad Pedagógica Nacional. Bogotá. 